|| Print ISSN: 2589-7837 || Online ISSN: 2581-3935 ||

International Journal of Medical Science and Diagnosis Research (IJMSDR)

Available Online at www.ijmsdr.com

NLM (National Library of Medicine ID: 101738824)

Original Research Article

Volume 6, Issue 1; January: 2022; Page No. 46-52

\title{
Rehabilitation of the Breached Tooth Fragment- A Minimal Invasive Cosmetic Approach: An Original Case Series
}

\section{Raji Viola Solomon ${ }^{1}$, Chavva Lakshmi Charan Reddy ${ }^{2}$, M.S. Rangareddy ${ }^{3}$, MD. Abdul Wahed ${ }^{4}$, Parasa Lohitha ${ }^{5}$}

${ }^{1}$ Professor, Department of Conservative Dentistry and Endodontics, Panineeya Institute of Dental Sciences and Research Centre, Hyderabad, Telangana, India.

${ }^{2}$ Senior Lecturer, Department of Conservative Dentistry and Endodontics, Panineeya Institute of Dental Sciences and Research Centre, Hyderabad, Telangana, India.

${ }^{3}$ Professor, Department of Conservative Dentistry and Endodontics, Panineeya Institute of Dental Sciences and Research Centre, Hyderabad, Telangana, India.

${ }^{4}$ Senior Lecturer, Department of Conservative Dentistry and Endodontics, Panineeya Institute of Dental Sciences and Research Centre, Hyderabad, Telangana, India.

${ }^{5}$ Post Graduate student, Department of Conservative Dentistry and Endodontics, Panineeya Institute of Dental Sciences and Research Centre, Hyderabad, Telangana, India

Conflicts of Interest: Nil

Corresponding author: Parasa Lohitha

DOI: https://doi.org/10.32553/ijmsdr.v6i1.904

\section{Abstract:}

Tooth fractures are often reported as emergencies in dental practice. Coronal fractures of the anterior teeth are a prevalent cause of dental trauma in children and teenagers. It's a tragic situation that necessitates prompt care and quick functional and esthetic repair. The clinician's main difficulty when dealing with these types of dental injuries is to re-establish the natural esthetics of the traumatized anterior tooth. There are several therapeutic options for this condition, one of which is reattachment of the fractured component itself. Because of its simplicity, natural esthetics, and preservation of tooth structure, reattachment of a tooth fragment should be the first choice and a feasible alternative to conventional approaches. For a successful prognosis, the patient's cooperation and comprehension of the treatment's limitations is essential. This paper presents a report of two clinical cases on managing complicated crown fractures, where the restoration of the fractured anterior teeth using the residual tooth fragment was supported by fiber post system.

Keywords: Complicated Crown Fracture, Fiber post, Fractured Tooth Fragment, Internal grooving.

\section{Introduction:}

Traumatic fractures of the anterior teeth are most frequent type of dental trauma in the permanent dentition. ${ }^{1}$ Because of their position and angulation in the dental arch, the maxillary central incisors are the most commonly affected teeth. Anterior teeth fractures require 
rapid treatment not only to restore function and appearance, but also because of the psychological impact on the patient. $^{2}$ Depending on the severity and extent of the fracture, the treatment choices available to the patient can range from a basic resin composite build up to a comprehensive restorative intervention. $^{3}$

Reattachment of the fractured fragment is one viable treatment modality for restoring a fractured anterior tooth. Only in circumstances where there is no or limited biological width violation and the fragment is retrieved in a substantially undamaged state could reattachment of the fractured fragment be considered. ${ }^{4}$ When compared to resin-based composite or a full-coverage crown, tooth fragment reattachment is a conservative, costeffective, cosmetic, functional, time-saving and has positive patient acceptance for restoring a fractured tooth. ${ }^{5}$ Fractured fragment reattachment provides good and long-lasting esthetics as the tooth's original anatomical form, colour, and surface texture are preserved. It not only restores the function but also has a positive emotional and psychological response from the patient due to the preservation of natural tooth structure. ${ }^{6}$

Due to advancements in adhesive techniques and restorative materials, it is now possible to reattach a fractured fragment. ${ }^{7}$ This article reports on management of two coronal tooth fracture cases, where the anterior teeth were successfully restored by the tooth fragment reattachment using fiber post and internal groove technique.

\section{Case Series}

\section{Case 1:}

A 25-year-old male patient reported to the department of Conservative Dentistry \& Endodontics with a chief complaint of pain and traumatic fracture of the upper right front teeth one day back. The patient had experienced trauma due a road traffic accident. The patient's medical history was non-contributory. Intraorally, clinical examination revealed a horizontal fracture in relation to 11 involving the middle of the clinical crown with associated pulp exposure without displacement of the fragment, as well as a horizontal fracture in relation to 12 with pulp exposure associated with missing fractured segment (Figure 1A). An intact periodontal ligament space, complete root formation, and no root fracture were observed on radiographic evaluation of both teeth. The periapical tissues and alveolar bone appeared to be in good condition. With respect to 11 and 12, a diagnosis of complicated crown fracture (involving the pulp chamber) - Ellis Class III was made (Figure 1B). The patient's treatment options included root canal therapy (RCT) followed by a post-core and crown or reattachment of the tooth fragment. The patient chose to have the tooth fragment reattached after examining the benefits, drawbacks, prognosis, and cost of each treatment option. As a result, it was decided to treat 11 with a single visit RCT and then reattach the same fragment with fibre post reinforcement. The procedure of RCT followed by a post-core restoration was scheduled for 12 .

Local anesthesia was administered (1.0 $\mathrm{cc}$ of lidocaine 2\% with 1: 80,000 epinephrine) and the fractured segment was atraumatically removed in relation to 11 (Figure 1C). It was then rinsed with $2 \%$ chlorhexidine solution and stored in isotonic saline solution to prevent dehydration and discoloration (Figure 1D). Isolation was achieved with a rubber dam and immediately root canal treatment was initiated and the working length was confirmed with radiograph using no. $15 \mathrm{k}$-file (Figure 1E). Chemo-mechanical preparation of the root canal was done followed by irrigation with $3 \%$ sodium hypochlorite and $17 \%$ EDTA solutions, and dried with absorbent paper points. A \#60 (2\%) master gutta-percha point was chosen, and sectional obturation was performed using endodontic resin sealer $(\mathrm{AH}$ plus, Dentsply), retaining approximately 4-5 
mm of gutta-percha apically (Figure $1 \mathrm{~F}$ and $\mathrm{G}$ ). Later appropriate fiber post size 1 (RelyX $^{\mathrm{TM}}$ Fiber Posts-3M ESPE) was selected (Figure $1 \mathrm{H})$. After post selection, the approximation of the fractured fragment was examined, and an internal groove was created on the fragment with a diamond fissured bur (ISO 173/014) (Figure 1I). Both fractured crown and root canal were etched with $37 \%$ orthophosphoric acid, rinsed, blot dried, followed by application of a dentin bonding agent (Tetric N-Bond Universal, Ivoclar Vivadent). Dual cure resin cement (Multilink N System - Ivoclar Vivadent) was placed in the canal followed by a fiber post up to proper length and the fragment was reattached to the tooth using flowable resin composite (Tetric N-Flow, Ivoclar Vivadent) (Figure 1J). Excess cement along the margins was scraped off and light cured for 30 seconds using a visible curing light. During the curing process, firm and consistent finger pressure was exerted, and care was taken to ensure that the fractured coronal fragment approximated accurately. The fracture line was examined and polished with polishing disks (Figure 1K). Root canal treatment was done for 12 followed by a fiber post and core and crown placement. The occlusion was evaluated, and postoperative instructions regarding prevention of loading on the anterior teeth were given to the patient. A one-year follow-up was performed, and no postoperative complications were reported (Figure 1L).

\section{Case 2:}

A 19-year-old male patient reported to the department of Conservative Dentistry \& Endodontics with the chief complaint of pain and fractured tooth in upper right front tooth region. A detailed history revealed that the patient had received a blow to the upper jaw 1 day prior, resulting in a fractured maxillary right central incisor. The patient recovered the fractured fragment at the site of injury and was brought to the clinic stored in water. Clinically, intraoral examination revealed an oblique fracture in the labiopalatal direction in relation to 11 which was above the gingival margin at cervical third of the crown, with the pulp involvement (complicated crown fracture Ellis Class III) (Figure 2A). The alveolar bone and periapical area seemed normal on radiographic evaluation, and there was no associated root fracture (Figure 2B). Upon examination of the fractured fragment, it was found that the fragment was in healthy condition and fit relatively well on the fractured tooth and was stored in saline solution (Figure 2C).

As the clinical scenario was similar to the first case, root canal treatment and reattachment of fractured fragment using fiber post were planned in a similar manner. Root canal treatment was initiated and working length was determined using no.15 k-file (Figure 2D). Chemo-mechanical preparation of the root canal was done followed by sectional obturation using resin based sealer (Figure 2E and F). Later appropriate size of fiber post was selected (Figure G) followed by placement of internal groove on the fragment (Figure 2H). After surface etching and bonding of both the fractured crown and root canal, dual cure resin cement was filled in the canal followed by placement of a fiber post, and the fragment was reattached with flowable resin composite similar to the above case (Figure 2I). Finishing and polishing was done using diamond burs and disks (Figure 2J). Occlusion was checked and postoperative instructions were given to the patient. No postoperative complications were found after a one year follow-up assessment (Figure 2K). 


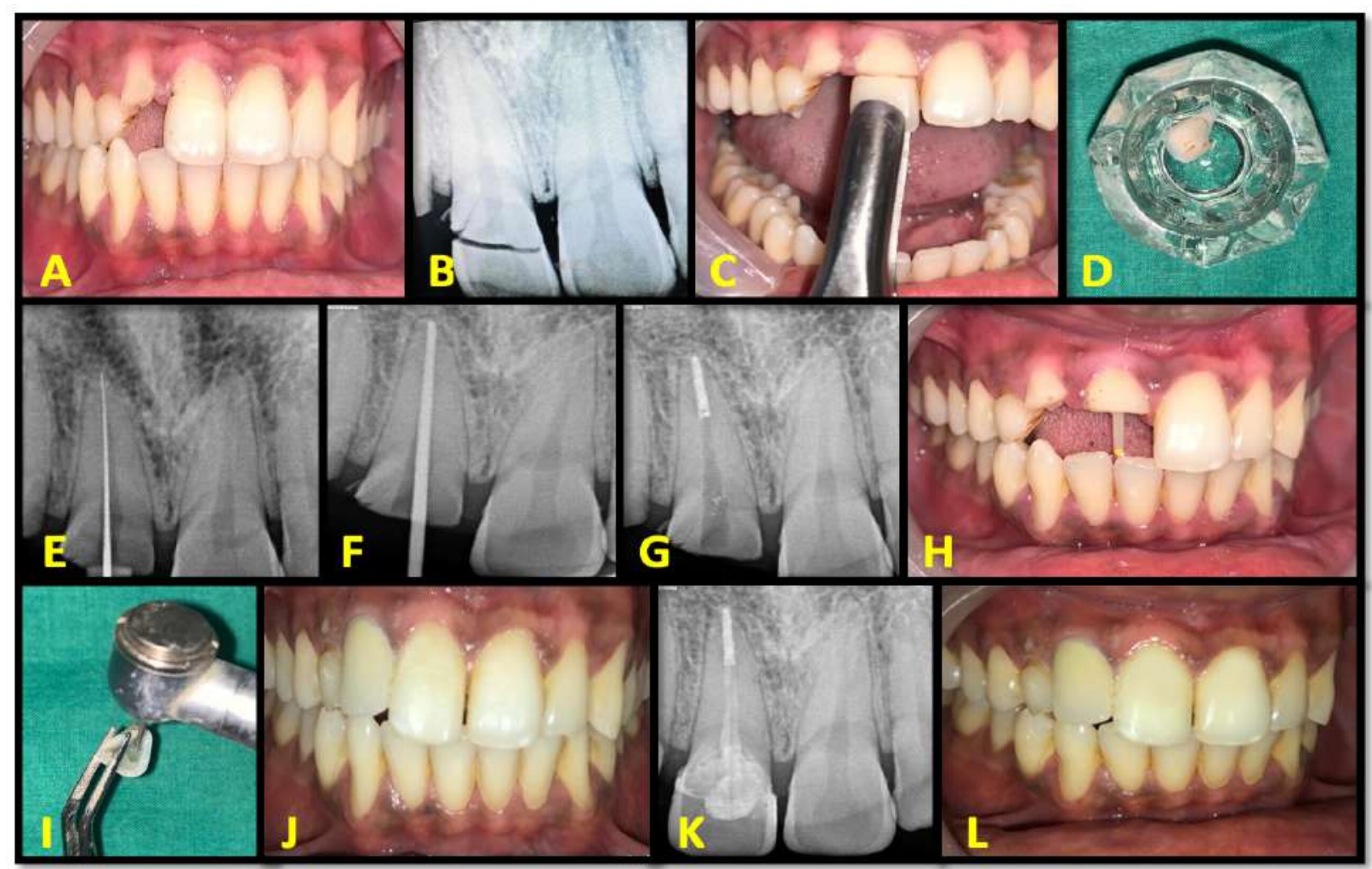

FIGURE 1: (A) Pre-operative clinical image irt 11 (B) Pre-operative radiographic image (C) Fractured fragment removal (D) Fractured fragment stored in saline solution (E) Working length determined (F) Master cone selection (G) Sectional obturation (H) Fiber post selection (I) Internal groove placement (J) Fractured fragment reattachment (K) Post-operative image (L) 1-year follow-up image

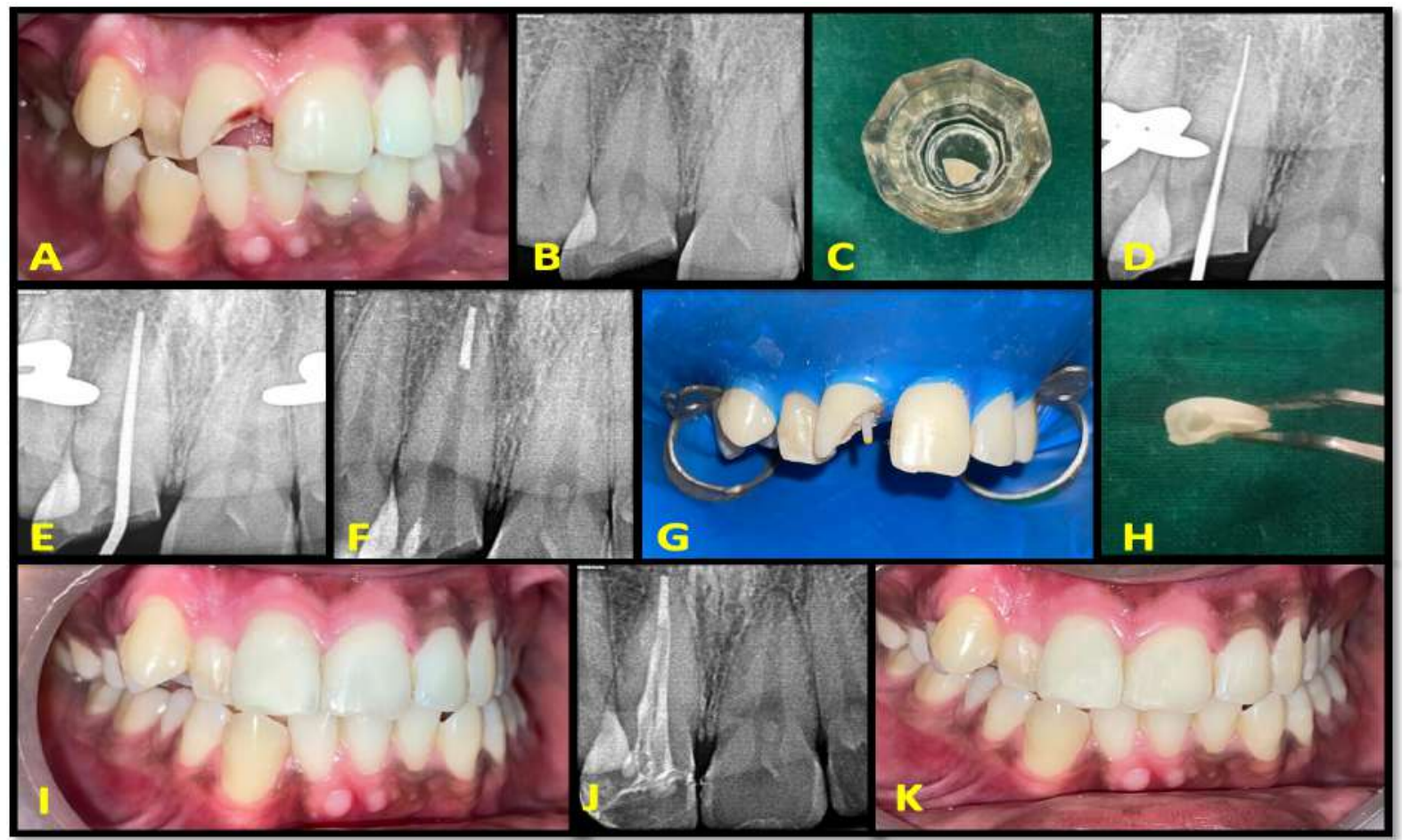

FIGURE 2: (A) Pre-operative clinical image irt 11 (B) Pre-operative radiographic image (C) Fractured fragment stored in saline solution (D) Working length determined (E) Master cone selection (F) Sectional obturation

(G) Fiber post selection (H) Internal groove placement (I) Fractured fragment reattachment (J) Post-operative image (K) 1-year follow-up image 


\section{Discussion}

Trauma to the anterior teeth is a relatively common incidence. Patients with fractured anterior teeth experience pain, fear, and emotional anxiety over their appearance. ${ }^{8}$ The optimum choice to offer these patients is usually an immediate restoration of the esthetic appearance while preserving the native tooth.

The factors influencing the success of such repairs are the location of the fracture, the size of the fracture remnants, pulpal involvement, periodontal health, biological width invasion, occlusion, mobility of remaining tooth, the operator's and patient's time and choice of adhesive material. ${ }^{9}$ In the case of reattachment, the extent of the fracture line is crucial. If the fracture line extends below the alveolar crest, orthodontic and surgical procedures are required to bring the fracture line within the biological limits. Gingivectomy is required if the fracture line is located above the alveolar crest but deep subgingivally, violating the biological width. However, these procedures were not required in the mentioned case reports since the fracture line did not encroach the biological width. ${ }^{10}$

Reattachment method was once considered a provisional therapy option for crown fractures, but with recent advancements, this approach now offers a promising prognosis. ${ }^{11}$ The success of reattaching uncomplicated tooth fractures has been documented in several case studies. When the fractured fragment is intact and available, fragment reattachment may be the most functional and esthetic treatment option, even in complicated fracture cases, as demonstrated in the present case reports. ${ }^{12}$

The reattachment strategies that have been suggested for reattaching a tooth fragment are circumferential bevel placement, simple reattachment, external chamfer placement, Vshaped enamel notch, internal groove placement and superficial over contour of restorative material over the fracture line. According to Reis et al (2001), fracture resistance of the attached fragment with simple reattachment accounts for $37.1 \%$, buccal chamfer for $60.6 \%$, over-contour for $97.2 \%$, and internal groove for $90.5 \%$, respectively. ${ }^{13}$ Greater extension of material on the surface in over contouring provides better force distribution but hampers the esthetic appearance. Internal grooving has thus been chosen for the above mentioned cases. In case of internal grooving, the resin flows into the groove and produces an internal resin bar that acts as an opponent to the compression load applied on the surface, resulting in improved mechanical outcomes. The precise fit between the fractured tooth fragment and the intact tooth is unaltered which helps to minimize the composite and enamel interface. ${ }^{13,14}$

Reattachment of the fragment, which is a minimally invasive procedure, may provide benefits such as improved esthetics due to perfect shade match and translucency, incisal edge wear at a rate equivalent to the adjacent teeth, and psychological benefit to the patient. ${ }^{9}$ According to research and clinical experience, reattaching fractured coronal fragments has demonstrated successful short and medium term results. ${ }^{15}$ Cavaller et al. found that reattachment of crown fracture had a better long-term prognosis than composite resin restorations. ${ }^{16}$ Yucel et al. evaluated the restored teeth using reattachment technique of fractured fragment to the remaining tooth and found a successful result after a 24-month follow-up. ${ }^{17}$

The success of an endodontically treated tooth is highly dependent on achieving a "fluid tight seal" by precisely and effectively performing an obturation. Sectional obturation was done in the above case reports, as it is ideal for postcore cases where only the apical portion of the canal needs to be filled. ${ }^{18}$

Modern adhesive techniques and materials have enabled the creation of monoblock effect, which is a multilayered structure without any weak inter-layer interfaces. Trope et al in 1985 
found that flowable resin composite restorations not only reinforced the tooth, but also aided in achieving greater bond strength of fractured segments. Additionally, the flowable composite minimizes air voids, reduces microleakage, and reinforces root strength. ${ }^{19}$

Fiber posts were utilised in this case series, because their elastic modulus is identical to that of the dentin, allowing for unidirectional flexing of the post and homogenous stress distribution. Furthermore, it requires minimal tooth preparation and reduces chair-side time. Also, using a fiber post in conjunction with self/light cured resin cement, as in the present case series, creates a monobloc effect, which protects the bond from rotational and twisting forces that may reduce the potential of success. ${ }^{20}$

The hydration of fracture fragments is essential because it aids in the retention of tooth colour and enhances the bond strength of the tooth. The literature is unclear on the time limit for dehydrated fragment. As per reports, complete rehydration of fragment takes 48 hours, so the fragment needs to be attached the next day. The fracture fragment was always hydrated in both of the cases discussed above. ${ }^{21}$

The longevity of the reattachment in the preceding case reports showed no separation of the crown fragment after one-year follow-up. To regulate esthetic, occlusion, pulpal, and periodontal outcomes, the tooth was assessed clinically and radiographically. There was no colour variation, resorption, or movement of the reattached fragment. The efficacy of this alternative treatment is aided by advancements in resin dental materials and techniques.

\section{Conclusion}

Under the limitations of present case series, it can be concluded that clinical and radiographic outcomes for reattaching the fractured tooth fragment were favorable and provides immediate esthetic benefits to the patient who has suffered dental trauma. Based on the aforementioned factors, the clinician must plan meticulously and possess a sound knowledge of materials and techniques. Fractured fragment reattachment could be the first choice for crown fractures of anterior teeth as it is the most conservative and less time consuming treatment option.

\section{References}

1. Dietschi D, Jacoby T, Dietschi JM, Schatz JP. Treatment of traumatic injuries in the front teeth: restorative aspects in crown fractures. Practical periodontics and aesthetic dentistry: PPAD. 2000 Oct 1;12(8):751-8.

2. Andreason JO, Andreason FM, Andreason L. Crownroot fractures. Textbook and color atlas of traumatic injuries to the teeth. 4th ed. Wiley Black well, 2007; 280- 90.

3. Chu FC, Yim TM, Wei SH. Clinical considerations for reattachment of tooth fragments. Quintessence Int 2000;31: 385-91.

4. Baratieri LN, Ritter AV, Monteiro Júnior $\mathrm{S}$, de Mello Filho JC. Tooth fragment reattachment: An alternative for restoration of fractured anterior teeth. Pract Periodontics Aesthet Dent 1998;10: 115-25.

5. Andreasen FM, Norén JG, Andreasen JO, Engelhardtsen S, Lindh-Stromberg U. Long-term survival of fragment bonding in the treatment of fractured crowns: A multicenter clinical study. Quintessence Int 1995;26:669-81.

6. Maia EA, Baratieri LN, de Andrada MA, Monteiro S Jr, de Araújo EM Jr. Tooth fragment reattachment: Fundamentals of the technique and two case reports. Quintessence Int 2003;34:99-107.

7. Baratieri LN, Monteiro S. Tooth fragment reattachment: Fundamentals of the technique and the casereport. Quintessence Int. 2003;34:99-107.

8. Hegede RJ. Tooth fragment reattachment an esthetic alternative: Report of a case. J 
Indian SocPedod Prev Dent. 2003;21:1179.

9. Darda SS, Manwar NU, Chandak MG, Shori DD (2008) Fracture reattachment: functional andaesthetic alternative: Case report. JFPA 22: 72-75.

10. Gurtu A, Roy S, Chandra P, Bansal R. Reattachment of complex fractures; a reality by advances in self-etch bonding systems. Indian Journal of Dental Research. 2019 Jan 1;30(1):135.

11. Tonini R. An innovative method for fragment reattachment after complicated crown fracture. Journal of Esthetic and Restorative Dentistry. 2017 May 6;29(3):172-7.

12. El Yamani A. Reattachment of a crown fragment: An immediate esthetic alternative. Periodontics. 2016;2(2):10.

13. Reis A, Francci C, Loguercio AD, Carrilho MR, Rodrigues Filho LE. Re-attachment of anterior fractured teeth: Fracture strength using different techniques. Oper Dent. 2001;26:287-94.

14. Kumar S, Maria R. Determining the Fracture Strength of the Reattached Fragment of Teeth: An In Vitro Study. Journal of Dental and Allied Sciences. 2013 Jan 1;2(1):16.

15. Attila OI, Cenk HM, Toroglu MS (2006) Multidisciplinary approach to the rehabilitation of a crown-root fracture with original fragment for immediate esthetics: a case report with 4-year follow-up.Dental Traumatology 22: 48-52.

16. Cavalleri G, German N (1995) Traumatic crown fractures in permanent incisors with immatureroots: a follow-up study. Endod Dent Traumatol11: 294-296.

17. Yucel Y, Cigdem Z, Ozge E, Nihal B (2008) Evaluation of success in the reattachment of coronalfractures. Dental Traumatology 24: 151-158.

18. Soumya S, Agarwal P, Patri G, Behera SS, Kumar M. Obturation an Overview. IP Indian Journal of Conservative and Endodontics. 2021 Mar 15;6(1):11-20.

19. Polesel A. Restoration of the endodontically treated posterior tooth. Giornale Italiano di Endodonzia. 2014 Jun 1;28(1):2-16.

20. Badami V, Reddy SK. Treatment of complicated crownroot fracture in a single visit by means of rebonding. J Am Dent Assoc 2011; 142(6):646-50.

21. Jyothi M, Jyothirmayi BS, Sirisha K, Mounika A, Girish K, Sruthi Keerthi MH. Reattachment-Conservative management of complicated crown fractures in anterior teeth. Int J Appl Dent Sci. 2016;2:10-3. 